\title{
Bone Cell Communication Factors Provide a New Therapeutic Strategy for Osteoporosis
}

\author{
Jung Ha Kim and Nacksung Kim* \\ Departments of Pharmacology, Chonnam National University Medical School, Hwasun, Korea
}

Bone homeostasis is strictly regulated by the balance between bone resorption by osteoclasts and bone formation by osteoblasts. Many studies have shown that osteoclasts affect osteoblasts, and vice versa, through diffusible paracrine factors, cell-cell contact, and cell-bone matrix interactions to achieve the correct balance between osteoclastic and osteoblastic activities in the basic multicellular unit (BMU). The strict regulation that occurs during bone remodeling hinders the long-term use of the currently available antiresorptive agents and anabolic agents for the treatment of osteoporosis. To overcome these limitations, it is necessary to develop novel agents that simultaneously inhibit bone resorption, promote bone formation, and decouple resorption from formation. Therefore, a more detailed understanding of the mechanisms involved in osteoclast-osteoblast communication during bone remodeling is necessary. Key Words: Osteoclasts; Osteoblasts; Cell Communication; Osteoporosis; Paracrine
Communication

This is an Open Access article distributed under the terms of the Creative Commons Attribution Non-Commercial License (http://creativecommons.org/licenses/by-nc/4.0) which permits unrestricted non-commercial use, distribution, and reproduction in any medium, provided the original work is properly cited.

\section{Article History:}

Received January 3, 2020

Revised January 23, 2020

Accepted January 23, 2020

\section{Corresponding Author:}

Nacksung Kim

Department of Pharmacology,

Chonnam National University Medical School, 264 Seoyang-ro,

Hwasun-eup, Hwasun 58128, Korea

Tel: +82-61-379-2835

Fax: +82-61-373-6974

E-mail: nacksung@jnu.ac.kr

\section{INTRODUCTION}

The bone is a dynamic organ that undergoes continuous renewal through bone remodeling processes to maintain its mechanical characteristics and calcium homeostasis. Bone remodeling is a complex and sophisticated series of sequential events, which occur within a temporary anatomical structure called the basic multicellular unit (BMU), involving various cell types including osteoclasts, osteoblasts, osteocytes, T-cells, macrophages, pericytes, vascular endothelial cells, canopy bone lining cells, and precursor populations of osteoblasts and osteoclasts. ${ }^{1-3}$ In particular, osteoclasts and osteoblasts are the two major cells regulating bone remodeling processes. Osteoclasts and osteoblasts are responsible for old bone resorption and new bone formation, respectively. Bone remodeling in each BMU proceeds in cycles consisting of distinct phases: the recruitment of osteoclasts and bone resorption by osteoclasts; the coupling of resorption to formation or reversal from catabolism to anabolism; the recruitment of osteoblasts and new bone formation by osteoblasts; and the termination of these processes. ${ }^{4-7}$ As an imbalance between bone formation and bone resorption results in multiple metabolic bone diseases like osteoporosis and osteopetrosis, each phase of the bone remodeling process must be strictly regulated by various local or systemic factors and intracellular signals to maintain bone homeostasis. ${ }^{8-11}$

Osteoporosis is the most common metabolic bone disease caused by excessive bone resorption relative to formation. It is characterized by low bone mass, the deterioration of bone tissue, and an increased risk of bone fracture. Osteoporosis-related fractures most commonly occur in the hip, wrist, spine, or shoulder, particularly in post-menopausal women. ${ }^{8-11}$

Several drugs are currently available for osteoporosis treatment. These drugs target either the inhibition of bone resorption or the promotion of bone formation. However, certain limitations of antiresorptive agents and boneforming drugs have been revealed. Antiresorptive drugs, such as alendronate, zoledronic acid, risedronate, and ibandronate, effectively block the formation and function of osteoclasts, but simultaneously reduce bone formation. In contrast, anabolic drugs, such as parathyroid hormones, teriparatide, and recombinant human parathyroid hormone, increase bone formation markers, but also increase bone resorption markers. These long-term adverse events 
induced by antiresorptives and bone-forming drugs suggest that the coupling process between bone resorption and formation plays a crucial role in the complete restoration of the bone removed during remodeling cycles. ${ }^{8,10-14}$ Therefore, an understanding of the signaling pathway involved in the coupling process will help develop novel drugs that simultaneously block bone resorption and promote bone formation without certain adverse events. Here, we have reviewed the coupling factors that may be an ideal target for the management of osteoporosis.

\section{RANKI/RANK SIGNALING}

The receptor activator of the nuclear factor kappa B ligand (RANKL) is an essential factor for osteoclast differentiation and function. It is secreted by osteoblasts and osteocytes, and binds to receptor activator of nuclear factor kappa B (RANK) on the surface of osteoclast precursors. ${ }^{3,15,16}$ In addition, the physiological roles of the RANKL in osteoblasts have recently been elucidated. The vesicular RANK, secreted from maturing osteoclasts, binds to the osteoblastic RANKL to promote bone formation by osteoblasts. The osteoblastic RANKL regulates bone formation through the activation of PI3K-Akt mTOR to induce the expression of runt-related transcription factor 2 (Runx2). ${ }^{3,17,18}$ Therefore, the RANKL-RANK system could regulate both bone resorption and bone formation by using RANKL forward signaling and RANKL reverse signaling, respectively.

Denosumab, a monoclonal antibody against RANKL, is available for the management of osteoporosis and skeletal problems caused by the spread of cancers to bone. Denosumab binds to RANKL, thereby inhibiting osteoclast forward signaling. Despite its efficacy in the inhibition of bone resorption, adverse effects, such as low bone formation, may impede long-term use. ${ }^{8,19-21}$ Interestingly, Ikebuchi and colleagues developed an anti-RANKL antibody that reduced osteoclast formation and function by binding and inactivating multiple RANKL monomers, and stimulated osteoblast differentiation by binding to the cell-surface of the RANKL. ${ }^{17,18}$ Therefore, RANKL-RANK forward or reverse signaling offers a new strategy for the management of osteoporosis, which is able to trigger bone formation while inhibiting bone resorption.

\section{SCLEROSTIN}

Sclerostin is encoded by the SOST gene in humans. ${ }^{22}$ After discovering that the lack of SOST expression was the cause of the high bone mass in human Van Buchem disease and sclerosteosis, considerable evidence from in vitro, animal, and human studies has demonstrated that sclerostin plays an important role in bone homeostasis. ${ }^{23,24}$ Sclerostin is secreted primarily from osteocytes, but not osteoblasts. $^{23,25}$ It has been identified as binding to LRP5/6 receptors and antagonizing the canonical Wnt pathway. ${ }^{26,27}$ The inhibition of the Wnt pathway by sclerostin leads to the inhibition of bone formation by osteoblasts. In addition, sclerostin stimulates bone resorption through its inhibitory action on the canonical Wnt pathway, because activation of the canonical Wnt pathway in osteoblasts increases the expression of osteoprotegrin (OPG), a decoy receptor for RANKL, and reduces bone resorption. ${ }^{14,24,28-30}$

Sclerostin expression is also detected in osteoclast precursors and its expression is decreased when osteoclasts are formed in vitro. ${ }^{24,31}$ Tnfrsf $11 \mathrm{~b}(\mathrm{Opg})-/$ - and Tnfsf 11 (Rankl)-transgenic mice with a high-bone turnover exhibited a low level of sclerostin, suggesting that the suppression of sclerostin was associated with bone resorption is critical for the coupling of bone resorption to formation. ${ }^{27,32}$

Romosozumab, a monoclonal antibody against sclerostin, can simultaneously increase bone formation and decrease bone resorption when administered subcutaneously. However, it is usually administered for only 1 year owing to its gradual decrease in efficacy, ${ }^{8,33-35}$

\section{SLIT3}

Slit guidance ligand (SLIT) proteins were originally identified as chemorepellents that controlled axon crossing in the midline of the brain. Recently, Kim et al ${ }^{36}$ reported that SLIT3 was a coupling factor to regulate resorption-formation coupling. SLIT3 production is increased during osteoclast differentiation. The secretion of SLIT3 by osteoclasts stimulates pre-osteoblast migration and $\beta$-cateninmediated osteoblast differentiation. In addition, SLIT3 suppresses osteoclast differentiation via the inhibition of Rac activation in an autocrine and paracrine manner. Therefore, the dual roles of SLIT3 in both osteoblasts and osteoclasts result in osteoporotic bone phenotypes that involve a decrease in bone formation and an increase of bone resorption in mice lacking Slit 3 or its receptor Robo $1 .^{36-38}$ Importantly, the injection of a truncated SLIT3 containing the ROBO-binding LRR2 domain into ovariectomized mice reversed ovariectomy-induced bone loss by simultaneously enhancing bone formation and reducing bone resorption. ${ }^{3,36-38}$

\section{SEMAPHORINS}

Although semaphorins (SEMAs) were first identified as axon guidance cues, they have been shown to play important roles in angiogenesis, tissue development, and the immune response ${ }^{39-42}$ Of the eight classes of semaphorin family proteins, several studies have suggested important roles of SEMA4D and SEMA3A in bone metabolism. ${ }^{3,43,44}$

SEMA4D is a transmembrane semaphorin highly expressed in osteoclasts, but not in osteoblasts. FC-SEMA4D, a soluble FC receptor SEMA4D fusion protein, inhibits osteoblast differentiation and function without altering proliferation. The binding of SEMA4D to its receptor complex, consisting of ErbB2 and Plexin-B1, leads to activation of the small GTPase RhoA. Genetically altered mice with Sema4d and Plxnb1 deletion, as well as mice expressing an 
osteoblast-targeted dominant-negative RhoA, exhibited a high bone mass due to enhanced osteoblastic bone formation. ${ }^{45,46}$ However, the regulation of bone mass by SEMA4D may be more complicated. Dacquin et al. ${ }^{44}$ reported that the increased bone mass phenotype in Sema4d-deficient mice was primarily due to a functional defect in osteoclasts. The authors showed that Sema4d-deficient primary osteoclasts led to delayed osteoclast differentiation and reduced osteoclast resorption activity that was in part due to the unbalanced regulation of $\beta 3$ integrin subunit signaling. ${ }^{44}$ Although the precise mechanisms through which SEMA4D contributes to bone homeostasis have not been elucidated, the injection of Sema4d siRNA or SEMA4Dspecific antibody into an ovariectomy-induced animal model of osteoporosis reversed bone mass, suggesting that SEMA4D was a beneficial target for osteoporosis treatment. $^{45,47}$

SEMA3A was first identified in the involvement of patterned neuronal connections and is now recognized as a mediator linking osteoclasts and osteoblasts. ${ }^{48}$ SEMA3A is mainly expressed by osteoblasts and its receptor, Nrp1, is expressed by osteoclast precursors. ${ }^{48-50}$ Sema3a-deficient osteoblasts showed a defect in osteoblast differentiation owing to the inhibition of $\beta$-catenin activation, whereas SEMA3A treatment caused a decrease in the differentiation of osteoclast precursors through the inhibition of RhoA activation. ${ }^{51}$ Hayashi et al.$^{51}$ reported that a global Sema3a deletion in mice caused a severe osteopenic phenotype that was associated with a decrease in osteoblastic bone formation and an increase in osteoclastic bone resorption. Interestingly, mice with osteoblast-specific deletion of Sema $3 a$ did not undergo any change in bone parameters, whereas mice with neuron-specific deletion of Sema3a exhibited a markedly low bone mass, similar to mice with global deletion of Sema $3 a{ }^{52}$ These results were indicative of the indirect effects of SEMA3A on bone metabolism through the nervous system. Furthermore, the injection of SEMA3A into ovariectomized mice prevented ovariectomyinduced bone loss, both through the promotion of bone formation and the suppression of bone resorption. ${ }^{51}$

\section{CTHRC1}

Collagen triple helix repeat containing 1 (CTHRC1) was originally identified in injured arteries. ${ }^{53}$ The expression of CTHRC1 was found to be induced in mature bone-resorbing osteoclasts. ${ }^{54}$ The recombinant CTHRC1 protein stimulated osteoblastic differentiation of marrow stromal ST2 cells. Cthrc1 null mice showed a lower bone mass due to decreased bone formation, whereas Cthrc1 transgenic mice exhibited a higher bone mass owing to an increase in bone formation. ${ }^{54}$ Collectively, evidence obtained from in vitro and in vivo experiments indicated that CTHRC1 was an important stimulator of osteoblastic bone formation. To further define whether CTHRC1 acted as a coupling factor, expressed only by mature bone-resorbing osteoclasts, to stimulate bone formation, recombinant RANKL was in- jected into mice with osteoclast-specific Cthrc1 deletion. The acute phase of osteoclastic bone resorption occurred to the same extent as in control mice, whereas the anabolic response followed by resorption was inhibited or delayed in the mice with osteoclast-specific deletion of Cthrc $1 .{ }^{54}$ In contrast, it has been shown that CTHRC1 was secreted by osteoblasts and some osteocytes, but not by osteoclasts. ${ }^{55}$ In that study, the authors also demonstrated that CTHRC1 negatively regulated osteoclast differentiation through the inhibition of RANKL-induced NF-kB signaling activation and ERK1/2 phosphorylation. Their results revealed that the lower bone mass observed in Cthrc1-null mice was also the result of increased bone resorption as well as a decreased bone formation. Collectively, the in vitro and in vivo evidence supports the potential importance of CTHRC1 in bone remodeling; however, it remains to be determined if the role of CTHRC1 in bone remodeling is mediated by signals from the osteoblast lineage or from osteoclasts.

\section{CONCLUSIONS}

Generally, coupling factors are the molecules that are involved in the stimulation of osteoblastic bone formation in response to osteoclastic bone resorption to preserve normal

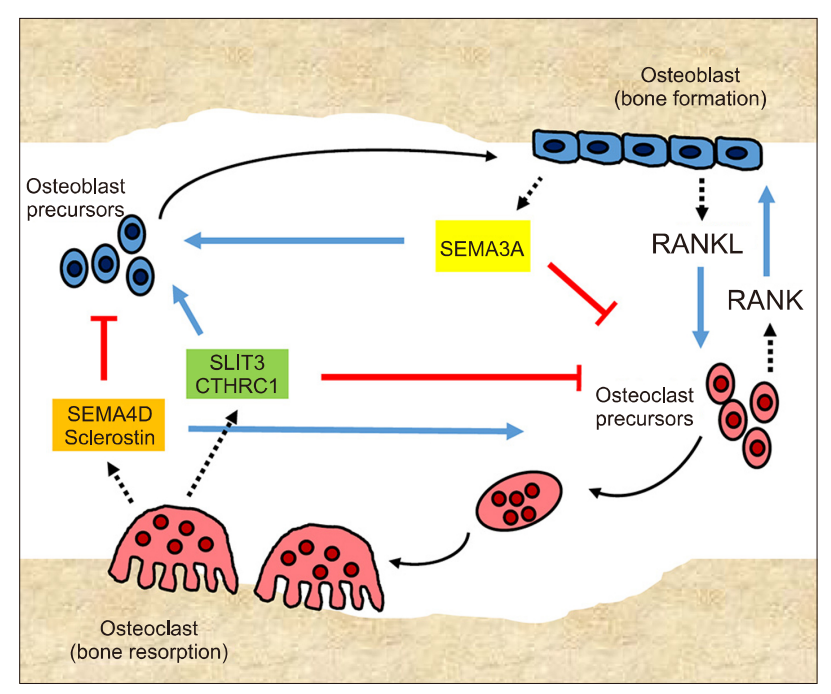

FiG. 1. The dual roles of bone cell communication factors during bone remodeling. The forward Receptor activator of nuclear factor kappa-B ligand (RANKL) signaling pathway originating from osteoblasts is known to induce osteoclast differentiation, and reverse RANKL signaling from osteoclasts also induces osteoblast formation. Several in vitro and in vivo studies have shown that some bone cell communication factors, such as semaphorin $3 \mathrm{~A}$ (SEMA3A), slit guidance ligand 3 (SLIT3), and collagen triple-helix repeat-containing 1 (CTHRC1), stimulate bone formation while suppressing bone resorption, and other factors, such as semaphorin 4D (SEMA4D) and sclerostin, inhibit bone formation while increasing bone formation. The roles of these bone cell communication factors in both osteoclasts and osteoblasts offer a new strategy for the development of bone disease therapies. 
bone mass. ${ }^{3,56}$ However, recent studies have shown that some molecules, such as sclerostin, SEMA4D, and SEMA3A, control bone remodeling through cell-cell communication between bone cells rather than a classical coupling process. Negishi-Koga et al. ${ }^{43,45}$ proposed that such factors should be called bone cell communication factors, as they participate in the bone remodeling process by regulating intercellular cross-talk among bone cells. ${ }^{3}$ Herein, we have discussed bone cell communication factors that are likely to be ideal therapeutic targets for osteoporosis (Fig. 1). As the orchestration of bone remodeling is strictly regulated by various known and as yet unknown bone communication factors, future investigations should be focused on the discovery of additional coupling signals and elucidate how these factors coordinate resorption and formation coupling in concert.

\section{ACKNOWLEDGEMENTS}

This work was supported by the National Research Foundation of Korea (NRF) grant funded by the Korea government (MSIT) (No. 2019R1A5A2027521).

\section{CONFLICT OF INTEREST STATEMENT}

None declared.

\section{REFERENCES}

1. Sims NA, Martin TJ. Coupling the activities of bone formation and resorption: a multitude of signals within the basic multicellular unit. Bonekey Rep 2014;3:481.

2. Sims NA, Walsh NC. Intercellular cross-talk among bone cells: new factors and pathways. Curr Osteoporos Rep 2012;10:109-17.

3. Kim BJ, Koh JM. Coupling factors involved in preserving bone balance. Cell Mol Life Sci 2019;76:1243-53.

4. Frost HM. The pathomechanics of osteoporoses. Clin Orthop Relat Res 1985;(200):198-225.

5. Matsuo K, Irie N. Osteoclast-osteoblast communication. Arch Biochem Biophys 2008;473:201-9.

6. Zaidi M. Skeletal remodeling in health and disease. Nat Med 2007;13:791-801.

7. Ikeda K, Takeshita S. Factors and mechanisms involved in the coupling from bone resorption to formation: how osteoclasts talk to osteoblasts. J Bone Metab 2014;21:163-7.

8. Chang B, Quan Q, Li Y, Qiu H, Peng J, Gu Y. Treatment of osteoporosis, with a focus on 2 monoclonal antibodies. Med Sci Monit 2018;24:8758-66.

9. Siris ES, Miller PD, Barrett-Connor E, Faulkner KG, Wehren LE, Abbott TA, et al. Identification and fracture outcomes of undiagnosed low bone mineral density in postmenopausal women: results from the National Osteoporosis Risk Assessment. JAMA 2001;286:2815-22.

10. Kanis JA, Cooper C, Rizzoli R, Reginster JY. European guidance for the diagnosis and management of osteoporosis in postmenopausal women. Osteoporos Int 2019;30:3-44.

11. Forciea MA, McLean RM, Qaseem A. Treatment of low bone den- sity or osteoporosis to prevent fractures in men and women. Ann Intern Med 2017;167:904.

12. Cotts KG, Cifu AS. Treatment of osteoporosis. JAMA 2018;319: 1040-1.

13. Rachner TD, Khosla S, Hofbauer LC. Osteoporosis: now and the future. Lancet 2011;377:1276-87.

14. Cosman F, de Beur SJ, LeBoff MS, Lewiecki EM, Tanner B, Randall S, et al. Clinician's guide to prevention and treatment of osteoporosis. Osteoporos Int 2014;25:2359-81.

15. Kong YY, Yoshida H, Sarosi I, Tan HL, Timms E, Capparelli C, et al. OPGL is a key regulator of osteoclastogenesis, lymphocyte development and lymph-node organogenesis. Nature 1999;397: 315-23.

16. Xiong J, Onal M, Jilka RL, Weinstein RS, Manolagas SC, O’Brien CA. Matrix-embedded cells control osteoclast formation. Nat Med 2011;17:1235-41.

17. Ikebuchi Y, Aoki S, Honma M, Hayashi M, Sugamori Y, Khan M, et al. Coupling of bone resorption and formation by RANKL reverse signalling. Nature 2018;561:195-200.

18. Zaidi M, Cardozo CP. Receptor becomes a ligand to control bone remodelling. Nature 2018;561:180-1.

19. McClung MR, Lewiecki EM, Cohen SB, Bolognese MA, Woodson GC, Moffett AH, et al. Denosumab in postmenopausal women with low bone mineral density. N Engl J Med 2006;354:821-31.

20. Leder BZ, Tsai JN, Uihlein AV, Wallace PM, Lee H, Neer RM, et al. Denosumab and teriparatide transitions in postmenopausal osteoporosis (the DATA-Switch study): extension of a randomised controlled trial. Lancet 2015;386:1147-55.

21. Bone HG, Wagman RB, Brandi ML, Brown JP, Chapurlat R, Cummings SR, et al. 10 years of denosumab treatment in postmenopausal women with osteoporosis: results from the phase 3 randomised FREEDOM trial and open-label extension. Lancet Diabetes Endocrinol 2017;5:513-23.

22. Balemans W, Ebeling M, Patel N, Van Hul E, Olson P, Dioszegi $\mathrm{M}$, et al. Increased bone density in sclerosteosis is due to the deficiency of a novel secreted protein (SOST). Hum Mol Genet 2001; 10:537-43.

23. Chen X, Wang Z, Duan N, Zhu G, Schwarz EM, Xie C. Osteoblastosteoclast interactions. Connect Tissue Res 2018;59:99-107.

24. Delgado-Calle J, Sato AY, Bellido T. Role and mechanism of action of sclerostin in bone. Bone 2017;96:29-37.

25. Winkler DG, Sutherland MK, Geoghegan JC, Yu C, Hayes T, Skonier JE, et al. Osteocyte control of bone formation via sclerostin, a novel BMP antagonist. EMBO J 2003;22:6267-76.

26. Li X, Zhang Y, Kang H, Liu W, Liu P, Zhang J, et al. Sclerostin binds to LRP5/6 and antagonizes canonical Wnt signaling. J Biol Chem 2005;280:19883-7.

27. Koide M, Kobayashi Y. Regulatory mechanisms of sclerostin expression during bone remodeling. J Bone Miner Metab 2019;37:917.

28. Glass DA 2nd, Bialek P, Ahn JD, Starbuck M, Patel MS, Clevers $\mathrm{H}$, et al. Canonical Wnt signaling in differentiated osteoblasts controls osteoclast differentiation. Dev Cell 2005;8:751-64.

29. Holmen SL, Zylstra CR, Mukherjee A, Sigler RE, Faugere MC, Bouxsein ML, et al. Essential role of beta-catenin in postnatal bone acquisition. J Biol Chem 2005;280:21162-8.

30. Kramer I, Halleux C, Keller H, Pegurri M, Gooi JH, Weber PB, 
et al. Osteocyte Wnt/beta-catenin signaling is required for normal bone homeostasis. Mol Cell Biol 2010;30:3071-85.

31. Pederson L, Ruan M, Westendorf JJ, Khosla S, Oursler MJ. Regulation of bone formation by osteoclasts involves Wnt/BMP signaling and the chemokine sphingosine-1-phosphate. Proc Natl Acad Sci U S A 2008;105:20764-9.

32. Koide M, Kobayashi Y, Yamashita T, Uehara S, Nakamura M, Hiraoka BY, et al. Bone formation is coupled to resorption via suppression of sclerostin expression by osteoclasts. J Bone Miner Res 2017;32:2074-86.

33. McClung MR, Grauer A, Boonen S, Bolognese MA, Brown JP, Diez-Perez A, et al. Romosozumab in postmenopausal women with low bone mineral density. N Engl J Med 2014;370:412-20.

34. Cosman F, Crittenden DB, Adachi JD, Binkley N, Czerwinski E, Ferrari S, et al. Romosozumab treatment in postmenopausal women with osteoporosis. N Engl J Med 2016;375:1532-43.

35. Kidd T, Bland KS, Goodman CS. Slit is the midline repellent for the robo receptor in Drosophila. Cell 1999;96:785-94.

36. Kim BJ, Lee YS, Lee SY, Baek WY, Choi YJ, Moon SA, et al. Osteoclast-secreted SLIT3 coordinates bone resorption and formation. J Clin Invest 2018;128:1429-41.

37. Koh JM. Osteoclast-derived SLIT3 is a coupling factor linking bone resorption to bone formation. BMB Rep 2018;51:263-4.

38. Iqbal J, Yuen T, Kim SM, Zaidi M. Opening windows for bone remodeling through a SLIT. J Clin Invest 2018;128:1255-7.

39. Castellani V, Rougon G. Control of semaphorin signaling. Curr Opin Neurobiol 2002;12:532-41.

40. Epstein JA, Aghajanian H, Singh MK. Semaphorin signaling in cardiovascular development. Cell Metab 2015;21:163-73.

41. Jongbloets BC, Pasterkamp RJ. Semaphorin signalling during development. Development 2014;141:3292-7.

42. Takamatsu H, Okuno T, Kumanogoh A. Regulation of immune cell responses by semaphorins and their receptors. Cell Mol Immunol 2010;7:83-8.

43. Negishi-Koga T, Takayanagi $H$. Bone cell communication factors and Semaphorins. Bonekey Rep 2012;1:183.

44. Dacquin R, Domenget C, Kumanogoh A, Kikutani H, Jurdic P, Machuca-Gayet I. Control of bone resorption by semaphorin 4D is dependent on ovarian function. PLoS One 2011;6:e26627.

45. Negishi-Koga T, Shinohara M, Komatsu N, Bito H, Kodama T,
Friedel RH, et al. Suppression of bone formation by osteoclastic expression of semaphorin 4D. Nat Med 2011;17:1473-80.

46. Han Y, You X, Xing W, Zhang Z, Zou W. Paracrine and endocrine actions of bone-the functions of secretory proteins from osteoblasts, osteocytes, and osteoclasts. Bone Res 2018;6:16.

47. Zhang Y, Wei L, Miron RJ, Shi B, Bian Z. Anabolic bone formation via a site-specific bone-targeting delivery system by interfering with semaphorin 4D expression. J Bone Miner Res 2015;30:28696.

48. Li Z, Hao J, Duan X, Wu N, Zhou Z, Yang F, et al. The role of Semaphorin 3A in bone remodeling. Front Cell Neurosci 2017;11: 40.

49. Koshihara Y1, Kodama S, Ishibashi H, Azuma Y, Ohta T, Karube S. Reversibility of alendronate-induced contraction in human osteoclast-like cells formed from bone marrow cells in culture. J Bone Miner Metab 1999;17:98-107.

50. Togari A, Mogi M, Arai M, Yamamoto S, Koshihara Y. Expression of mRNA for axon guidance molecules, such as semaphorin-III, netrins and neurotrophins, in human osteoblasts and osteoclasts. Brain Res 2000;878:204-9.

51. Hayashi M, Nakashima T, Taniguchi M, Kodama T, Kumanogoh A, Takayanagi H. Osteoprotection by semaphorin 3A. Nature 2012;485:69-74.

52. Fukuda T, Takeda S, Xu R, Ochi H, Sunamura S, Sato T, et al. Sema3A regulates bone-mass accrual through sensory innervations. Nature 2013;497:490-3.

53. Pyagay P, Heroult M, Wang Q, Lehnert W, Belden J, Liaw L, et al. Collagen triple helix repeat containing 1 , a novel secreted protein in injured and diseased arteries, inhibits collagen expression and promotes cell migration. Circ Res 2005;96:261-8.

54. Takeshita S, Fumoto T, Matsuoka K, Park KA, Aburatani H, Kato $\mathrm{S}$, et al. Osteoclast-secreted CTHRC1 in the coupling of bone resorption to formation. J Clin Invest 2013;123:3914-24.

55. Jin YR, Stohn JP, Wang Q, Nagano K, Baron R, Bouxsein ML, et al. Inhibition of osteoclast differentiation and collagen antibody-induced arthritis by CTHRC1. Bone 2017;97:153-67.

56. Delaisse JM. The reversal phase of the bone-remodeling cycle: cellular prerequisites for coupling resorption and formation. Bonekey Rep 2014;3:561. 